\title{
A importância do controle de qualidade nos laboratórios de análises clínicas - uma revisão integrativa
}

\author{
The importance of quality control in clinical analysis laboratories - an integrative review
}

\author{
Kariny Alves Santos" ${ }^{\circ}$, Marcio Trevisan ${ }^{\bullet}$ \\ Faculdade de Palmas, Palmas, Tocantins, Brasil. *Autor para correspondência. E-mail: kariny.alves6@gmail.com
}

\begin{abstract}
Resumo: Introdução: Os laboratórios de análises clinicas modernos são parte importante do sistema de saúde, pois resultados laboratoriais são responsáveis por até $75 \%$ das informações necessárias para a decisão do tratamento medicamentoso. Assim, esta pesquisa, através de uma revisão bibliográfica, buscou demonstrar qual a importância da implantação e manutenção do controle de qualidade em um laboratório de análises clínicas. Revisão: Trata-se de uma revisão de literatura do tipo integrativa. O levantamento de dados científicos e a sistematização das informações são oriundas de produções científicas publicadas de 2011 a 2021, no idioma português, indexadas na base de dados da BVS, MEDLINE-PUBMED, LILACS e SCIELO. Discussão: A necessidade de se implementar padrões de controle e gestão da qualidade em serviços de assistência à saúde tem apresentado um significativo crescimento nos últimos anos. A implantação de um sistema de controle de qualidade pode resultar em diversos benefícios, como redução de custos e desperdícios, aumento dos lucros laboratoriais, aumento da competitividade do laboratório e o estabelecimento da confiança do negócio no mercado. Considerações finais: Embora haja uma transformação tecnológica ocorrida no meio laboratorial, os erros ainda acontecem. A principal fonte de erros continua sendo oriunda da atuação do ser humano, o que reforça a necessidade da implantação dos controles de qualidade nas diferentes fases da análise laboratorial com o objetivo de diminuir essa interferência, diminuindo custos oriundos de retrabalhos e, consequentemente, aumentando a credibilidade do serviço.
\end{abstract}

Palavras-chave: controle de qualidade, norma PALC, qualidade laboratorial.

\begin{abstract}
Introduction: Modern clinical analysis laboratories are an important part of the health system, as laboratory results are responsible for up to $75 \%$ of the information needed to decide on drug treatment. Thus, this research, through a bibliographic review, sought to demonstrate the importance of implementing and maintaining quality control in a clinical analysis laboratory. Review: This is an integrative literature review. The collection of scientific data and the systematization of information come from scientific productions published from 2011 to 2021, in Portuguese, indexed in the database of the BVS, MEDLINEPUBMED, LILACS and SCIELO. Discussion: The need to implement quality control and management standards in health care services has shown significant growth in recent years. The implementation of a quality control system can result in several benefits, such as reduced costs and waste, increased laboratory profits, increased laboratory competitiveness and the establishment of business confidence in the market. Final considerations: Although there is a technological transformation that has taken place in the laboratory environment, errors still happen. The main source of errors continues to come from human activities, which reinforces the need to implement quality controls in the different phases of laboratory analysis in order to reduce this interference, reducing costs arising from rework and, consequently, increasing the service credibility.
\end{abstract}

Keywords: quality control, PALC standard, laboratory quality.

\section{Introdução}

Os laboratórios de análises clinicas modernos são parte importante do sistema de saúde, contribuindo diretamente para ações de prevenção, diagnóstico, tratamento e gerenciamento de patologias (Shcolnik et al., 2019; Codagnone et al., 2014). Os laboratórios clínicos envolvem processos e técnicas para realização dos exames laboratoriais que auxiliam no diagnóstico clínico contribuindo para que o médico estabeleça uma melhor conduta terapêutica. A participação de um laboratório no diagnóstico é um processo iniciado com a coleta do espécime diagnóstico e termina com a emissão de um laudo (Codagnone \& Guedes, 2014).

Os resultados das análises laboratoriais são responsáveis por até $75 \%$ das informações necessárias para a escolha de qual caminho seguir no tratamento medicamentoso. Com os avanços tecnológicos na medicina 
laboratorial, o desafio é prestar um atendimento humanizado, com taxa de produtividade alta e baixo custo. Dessa forma, faz-se necessário a implantação de um sistema de qualidade visando à padronização dos processos, otimização dos custos, melhoria nas análises laboratoriais e a satisfação dos clientes (Barbosa \& Mansour, 2019).

O controle de qualidade em um laboratório clínico é a prática de auditoria interna e externa, que objetiva assegurar que os resultados qualitativos e quantitativos sejam completos e sem erros, nas quais os auditores observarão os manuais, procedimentos, fluxogramas, políticas internas e capacidade de rastrear erros. $\mathrm{O}$ programa de controle da qualidade é um conjunto de ações que visam garantir a reprodução e a exatidão dos resultados dos exames, empregando a verificação dos parâmetros que continuamente intervêm no processo, melhorando-o. Este deve ser constante e permanente em todas as atividades do laboratório (Pasquini, 2018).

A importância de implantar um sistema de controle de qualidade no laboratório de análises clinica é reconhecer e diminuir os erros analíticos no laboratório, de forma que seja possível avaliar o desempenho do mesmo e consequentemente obtendo resultados confiáveis e seguros. Todo laboratório de análises clinicas que deseja implantar um sistema de qualidade eficiente, necessariamente deve providenciar uma infraestrutura adequada, uma equipe técnica selecionada e treinada, reagentes de qualidade, sistemas de limpeza corretos, processos de coleta e armazenamento de amostras de acordo com a metodologia implantada, e manutenções periódicas dos equipamentos. Para implantação do controle de qualidade laboratorial a gerência deve seguir alguns preceitos essenciais, começando pela aquisição de amostras de controle e conscientização dos funcionários (Santos et al., 2020).

O controle de qualidade é elaborado sempre através de algum tipo de análise ou medição e suas principais vantagens são mensuradas através da otimização de todo o processo, padronização de procedimentos, redução de tempo e de desperdícios, aumento do grau de certeza da qualidade, dos insumos utilizados e dos produtos finais (Manso \& Seabra, 2020). Durante a realização de um exame laboratorial, alguns fatores são necessários para satisfazer as necessidades dos clientes e permitir a verificação correta dos resultados. Por esse motivo, o controle de qualidade de laboratórios clínicos é de grande relevância, pois além de aumentar a segurança, traz credibilidade para o laboratório, favorecendo assim a liberação de laudos confiáveis (Kaim et al., 2019).

Nos laboratórios clínicos a busca incessante por melhorar os processos passou a ser uma meta a ser alcançada e seguida. A ciência médica e a tecnologia laboratorial vêm avançando rapidamente na história da assistência à saúde. Antigamente, a maioria dos laboratórios clínicos realizavam, rotineiramente, apenas cerca de 20 tipos de exames. Hoje, laboratórios clínicos realizam centenas de tipos de exames, alguns destes, em casos de emergência e urgência (Manso \& Seabra, 2020).

Alcançar a acreditação de laboratórios de análises clínicas exige o cumprimento de requisitos internacionais de qualidade, e este deveria ser um objetivo organizacional de todos os laboratórios de análises clínicas. Porém, é uma tarefa árdua e cara, no entanto, com vantagens que podem ser revertidas em aumento dos lucros do empreendimento e satisfação dos clientes (Oliveira \& Melo, 2020).

A satisfação do cliente é algo que recebe cada vez mais atenção durante a realização de testes de laboratório. Portanto, o controle de qualidade em laboratórios de análises clínicas é muito importante para melhorar a credibilidade e segurança para os pacientes, promovendo a rastreabilidade e a organização de dados corretos e confiáveis, evitando erros repetitivos no trabalho e garantir eficiência e qualidade na análise laboratorial.

Assim, esta pesquisa, através de uma revisão bibliográfica, buscou demonstrar qual a importância da implantação e manutenção do controle de qualidade em um laboratório de análises clínicas. Ressaltasse que esta revisão busca apresentar a importância do controle de qualidade, resguardando para trabalhos futuros a apresentação dos tipos de controles de qualidade existentes. A relevância do tema é assegurada quando Santos et al. (2020), verificaram que cerca $12.5 \%$ dos erros laboratoriais podem ter efeitos sobre a saúde dos pacientes. Por fim, esta pesquisa visa contribuir com profissionais da área e estudantes, considerando que existem poucas publicações sobre o tema, uma vez que reúne informações que poderão ser utilizadas como fonte de pesquisa futura.

\section{Revisão}

Esta pesquisa trata-se de uma revisão bibliográfica de natureza exploratória, através de uma revisão de literatura do tipo integrativa. O levantamento de dados científicos e a sistematização das informações são oriundas de produções científicas publicadas de 2011 a 2021, no idioma português, indexadas na base de dados da Biblioteca Virtual em Saúde (BVS), Literatura Internacional em Ciências da Saúde (MEDLINE-PUBMED), 
Literatura Latino Americana do Caribe em Ciências da Saúde (LILACS) e Scientific Electronic Library Online (SCIELO). Os descritores utilizados foram: controle de qualidade, norma PALC e qualidade laboratorial.

Laboratórios de análises clínicas são definidos como um serviço destinado a analisar amostras de paciente, objetivando oferecer apoio ao diagnóstico e terapêutico, compreendendo as fases pré-analítica, analítica e pós-analítica (Santos \& Junior, 2015). Esses laboratórios têm um papel importante no diagnóstico, controle e prevenção de doenças em todo o mundo. Os serviços que eles prestam contribuem para a contenção do surto de doenças e a detecção de novos patógenos. No entanto, isso depende da capacidade técnica dos laboratórios clínicos e principalmente da qualidade de seus serviços (Oliveira \& Melo, 2020; Silva \& Steckert, 2019).

Quando se verifica a importância das análises clínicas para a decisão diagnóstica e as condutas terapêuticas, é notória a importância e a necessidade de garantias da normalidade sanitária nos estabelecimentos clínicos e de mecanismos de controle e promoção da qualidade em todo o ciclo laboratorial (Trevisan, 2017). Como para outros setores da economia a implantação de controles de qualidade nos serviços laboratoriais é uma condição definitiva. É possível definir qualidade, no âmbito empresarial, como a capacidade de atender as necessidades e expectativas do cliente (Garrido \& Araújo, 2014). O processo de produção e a oferta dos serviços de saúde têm o controle de qualidade como fator imprescindível para atender a expectativa e a satisfação dos clientes (Castelo Branco et al., 2014).

Quando se pensa em qualidade dos resultados e nas etapas do laboratório clínico, é fundamental estabelecer padrões e procedimentos para prevenir e detectar os possíveis erros que ocorrem desde o pedido até a entrega do laudo. Identificar essas falhas é fundamental para adoção de medidas corretivas e preventivas como parte do controle de qualidade (Silva \& Steckert, 2019).

A execução de exames laboratoriais é dividida em três etapas: pré-analítica que consiste no preparo do paciente, identificação, coleta, manipulação e armazenamento da amostra biológica, ou seja, todas as atividades que antecedem as análises laboratoriais; analítica que é a realização dos testes e a interpretação dos resultados, onde os métodos utilizados, antes de serem implantados na rotina, são analisados em relação ao tipo da amostra, duração do ensaio, precisão, exatidão, sensibilidade, especificidade, linearidade, estabilidade dos reagentes, garantindo confiabilidade dos resultados e a última fase, pós-Analítica que se inicia com resultado quantitativo e/ou qualitativo obtido após as análises e é finalizada com a entrega do laudo (Barbosa \& Mansour, 2019).

Nesse sentido de busca constante pela qualidade, foi criado o Programa de Acreditação de Laboratórios Clínicos, popularmente conhecido como norma PALC, pela Sociedade Brasileira de Patologia Clínica/Medicina Laboratorial - SBPC/ML. Esta norma possui uma série de exigências que objetivam garantir a qualidade dos laboratórios por meio da aplicação de práticas e padrões que melhorem o desempenho. Ela está dividida em 17 categorias que definem exigências específicas de qualidade para todas as áreas de um laboratório de análises clínicas (Pasquini, 2018). O PALC-SBPC/ML possui 22 anos de implantação e vem cumprindo sua missão de promover a qualidade dos serviços laboratoriais prestados a pacientes e usuários, zelando pelo cumprimento de requisitos da qualidade, colaborando para uma adoção da cultura de melhoria continua na prática diária dos laboratórios clínicos brasileiros (SBPC/ML, 2021).

A PALC é a norma que melhor se adequa aos laboratórios de análises clínicas que atendem pacientes baseando-se nos seus critérios mais técnicos e menos processuais quanto à coleta e conservação das amostras, atendimento aos clientes, qualidade das análises, cumprimento de prazos e cuidados com resultados. Essa norma determina a revisão e o fluxo de seus processos para torná-lo mais confiável e seguro, melhorando o desempenho do laboratório e a confiança de seus resultados (Barbosa \& Mansour, 2019).

Essa norma prioriza a organização geral do laboratório, documentações necessárias para registro dos processos realizados, atendimento ao cliente desde o cadastro até a identificação da amostra, garantia dos métodos, reagentes e equipamentos usados na realização de exames, dos processos analíticos com base nos controles de qualidade interno e externo, garantindo resultados confiáveis e fidedignos, garantindo qualidade e confiabilidade dos resultados e laudos emitidos. Em suma, essa é uma norma específica e abrangente, com exigências que incluem todas as áreas críticas do laboratório clínico e as fases pré-analítica, analítica e pósanalítica (SBPC/ML, 2021).

É importante um controle de qualidade nos laboratórios clínicos, pois a liberação de um laudo com informações equivocadas pode contribuir com aumento de consultas médicas e testes laboratoriais e de imagem, elevando ainda mais o custo dos serviços de saúde (Vieira et al., 2011). Na área laboratorial, assim como em qualquer outra atividade, é praticamente impossível eliminar completamente os erros, mas é possível reduzi-los através de controles de qualidade (Guimarães et al., 2011).

Erros laboratoriais podem ter diferentes consequências sobre a saúde dos pacientes. Estes erros variam de acordo com o tipo de laboratório, os tipos de exames realizados e o tipo de paciente atendido. Mais de $75 \%$ 
dos erros geram resultados ainda dentro dos intervalos de referência; $12.5 \%$ levam a resultados errados ou incoerentes, fazendo com que os mesmos não sejam considerados clinicamente pelos médicos; e 12.5\% dos erros laboratoriais podem ter efeitos sobre a saúde dos pacientes. Assim, é indispensável minimizar tais erros, sendo necessário implantar um sistema de controle de qualidade a fim de verificar todos os processos da empresa e como esses os mesmos podem interferir com a melhoria da qualidade dos produtos e serviços referente aos clientes (Santos et al., 2020).

Shcolnik et al. (2020) levantaram que erros nos resultados de exames laboratoriais são observados com frequência, e que por volta de $20 \%$ dessas falhas resultam em danos aos pacientes. Assim, um programa de controle qualidade deve ser mantido para detectar possíveis falhas, reais ou potenciais; agir corretivamente sobre falhas e, quando possível, preventivamente antes que elas ocorram e provoquem impactos indesejados para os clientes; investigar a causa raiz das falhas e implantar ações que eliminem definitivamente essas falhas dos processos, evitando sua recorrência (Pasquini, 2018). Laboratórios clínicos devem aprimorar o sistema de controle de qualidade com o uso de procedimentos padronizados, condizentes com regulamentações técnicas e boas práticas de laboratório, a fim de garantir a confiabilidade dos resultados com o mínimo de erros e interferências e a integridade das pessoas, instalações e equipamentos (Dias et al., 2017).

Para atingir a qualidade do produto final é preciso que se faça padronização de todo o processo laboratorial que envolve desde a solicitação médica do exame até a liberação do laudo, para isso, é necessário responsabilidade e comprometimento de todos os profissionais de saúde envolvidos nessas etapas. $\mathrm{O}$ laboratório clínico deve estabelecer um sistema de controle da qualidade adequado ao tipo, à diversidade e ao volume de trabalho realizado. Tudo precisa ser documentado, e esta documentação deve estar disponível para o uso dos profissionais envolvidos. O manual da qualidade e a respectiva documentação da qualidade devem declarar a política do laboratório e os procedimentos operacionais padrões estabelecidos para satisfazer os requisitos da qualidade (Manso \& Seabra, 2020).

O exame laboratorial ocupa uma posição importante e crescente no processo de diagnóstico e monitoramento dos efeitos do tratamento médico moderno. As estatísticas mostram que cerca de dois terços das decisões clínicas importantes sobre admissão e alta são baseadas em informações contidas em testes de laboratório. O laboratório clínico deve garantir que os resultados produzidos reflitam de maneira confiável e consistente a situação clínica apresentada pelo paciente e não representem nenhum resultado interferente durante a análise. As informações geradas devem atender às necessidades de seus clientes, sendo capazes de determinar e realizar corretamente o diagnóstico, tratamento e prognóstico da doença (Manso \& Seabra, 2020).

A partir da Resolução de Diretoria Colegiada RDC302/2015, o controle interno de qualidade, passou a ser indispensável se tornando um requisito para o funcionamento dos laboratórios clínicos. É realizado diariamente junto à rotina na fase analítica, envolvendo a reprodutibilidade e a precisão de cada processo, visando melhorar a qualidade e identificar possíveis falhas, como o desvio do desempenho das análises, estabilidade de reagente e calibradores (Santos et al., 2020). É importante a realização do controle de qualidade em laboratórios de análises clínicas, para assegurar a qualidade e segurança dos exames, garantindo ao paciente um resultado seguro, eficaz, com informações fidedignas e de qualidade (Santos \& Junior, 2015).

\section{Discussão}

A qualidade, independente do ramo em que se atua, não pode ser mais vista pelas organizações apenas como um diferencial, mas também, como um mecanismo para prognosticar problemas, evitando que ocorram, e, em último caso, se ocorrerem, deve-se solucioná-los. O capitalismo, nos dias de hoje, propiciou um cenário cheio de novidades, novos produtos, marcas e concorrentes, as ferramentas dessa área empresarial auxiliam na estruturação de estratégias fundamentais para manutenção e fortificação do elo com clientes e consumidores finais. Consequentemente, dentre as várias vantagens dessa aplicação, ocorrem o aumento de vendas e da competitividade, consolidação da imagem e fidelização de clientes (Paula et al., 2017).

A necessidade de se implementar padrões de controle e gestão da qualidade em serviços de assistência à saúde tem apresentado um significativo crescimento nos últimos anos, caracterizando-se como um aspecto de extrema importância para organizações que atuam neste setor. Existem exigências maiores em termos de atendimento da legislação vigente, adequada gestão de custos e verificação de qualidade pelos usuários destes serviços (Berssaneti et al., 2016). 
Na visão de Santos et al. (2020) para se obter resultados confiáveis e seguros, os gestores dos laboratórios de análises clinicas, devem implantar um sistema de controle da qualidade que permita aos seus integrantes a garantia dos resultados diariamente, seguindo alguns passos como: tomar providências imediatas para eliminar as causas das não conformidades encontradas, através de ações corretivas; tomando medidas preventivas para evitar uma nova ocorrência das não conformidades encontradas. Já para Souza et al. (2020) a falta de um controle de qualidade eficiente provocará erros na fase pré-analítica, culminando em perdas de dados epidemiológicos de grande relevância para a saúde do paciente, gastos desnecessários com acondicionamento, transporte e posterior descarte das amostras biológicas não conformes e não processadas, além de transtornos e gastos adicionais com a busca de pacientes para nova coleta, quando necessário.

A implantação de um sistema de controle de qualidade pode resultar em diversos benefícios, tais como redução de custos e desperdícios, aumento dos lucros laboratoriais, aumento da competitividade do laboratório e o estabelecimento da confiança do negócio no mercado. No entanto, o maior benefício que se alcança quando se faz a gestão de qualidade dos processos de um laboratório, é o aumento da segurança oferecida aos pacientes e a satisfação dos clientes (Oliveira \& Melo, 2020).

\section{Considerações finais}

A busca por um aumento da qualidade e alta competitividade no mercado, faz com que a necessidade de um controle de qualidade nas empresas seja cada vez mais necessária, entretanto essa busca não deve se limitar apenas à conferência do produto final, e sim todo o processo precisa ser analisado para resultar no aumento da qualidade. Laboratórios de análises clínicas estão sujeitos ao cometimento erros ao longo de toda a cadeia de realização de um exame, uma vez que ele depende da ação humana. No entanto, é importante ressaltar que o cumprimento das especificações contidas nos procedimentos de controle de qualidade pode minimizar ou acabar com os erros oriundos nas etapas em que envolvam o fator humano. Programas de educação continuada e treinamentos periódicos com toda a equipe e auditorias internas, podem garantir a manutenção da execução do processo com excelência e minimização de erros e falhas.

O que se pode observar na bibliografia consultada é que, embora haja uma transformação tecnológica ocorrida no meio laboratorial, os erros ainda acontecem. A principal origem de erros continua sendo oriunda da atuação humana, o que reforça a necessidade da implantação dos controles de qualidade nas diferentes fases da análise laboratorial com o objetivo de diminuir essa interferência, diminuindo custos oriundos de retrabalhos e, consequentemente, aumentando a credibilidade do serviço.

Por fim, verificou-se que existem poucas publicações sobre o escopo desta pesquisa, e que apesar de sua importância, é um assunto pouco falado no meio acadêmico.

\section{Referências}

Barbosa, L. O., \& Mansour, S. N. 2019. Projeto de implantação da gestão da qualidade com base na norma PALC e metodologia ONA em um laboratório de análises clínicas. Revista Brasileira de Análises Clínicas, 50(4), 365-370.

Berssaneti, F. T., Saut, A. M., Barakat, M.F, \& Calarge, F. A. 2016. Existe uma relação entre os programas de acreditação e os modelos de excelência organizacional? Revista da Escola de Enfermagem da USP, $50(4), 650-657$.

Castelo Branco, N. M., Romão, C. M. C. P. A., Fialho, M. M. \& Delgado, I. F. 2014. Pesquis a de Satisfação: o olhar dos clientes sobre os laboratórios do Instituto Nacional de Controle da Qualidade em Saúde. Revista Vigilância Sanitária em Debate: Sociedade, Ciência \& Tecnologia, 2(1), 75-80.

Codagnone, F. T., Alencar, S. M. F., Shcolnik, W., Chaves, S. R. S., Silva, L. A., Henriques, V. H. O., \& Spitz. 2014. O uso de indicadores da fase pré-analítica como ferramenta da gestão laboratorial. Jornal Brasileiro de Patologia e Medicina Laboratorial, 50(2), 100-104.

Codagnone, F. T., \& Guedes, S. S. 2014. Buscando a eficiência laboratorial por meio de indicadores de qualidade: ênfase na fase pré-analítica. Revista Acreditação, 4(8), 27 - 41.

Dias, V. S., Barquette, F. R. S., \& Bello, A. R. 2017. Padronização da qualidade: alinhando melhorias contínuas nos laboratórios de análises clínicas. Revista Brasileira de Análises Clínicas, 49(2), 164-169. 
Garrido, R. G., \& Araujo, K. 2014. Sistemas de Gestão da Qualidade em Laboratório de Genética Forense. Revista Espacios, 35(5), 3-8.

Guimarães, A. C., Wolfart, M., Brisolara, M. L., \& Dani, C. 2011. O laboratório clínico e os erros préanalíticos. Revista do Hospital das Clínicas de Porto Alegre, 31(1), 66-72.

Kaim, M., Fischer, V. B., \& Comparsi, B. 2019. Ações de melhoria do sistema de gestão da qualidade do laboratório clínico escola de biomedicina. Revista saúde integrada, 12(24), 108-125.

Manso, J. R., \& Seabra, O. 2020. A importância da qualidade na etapa pré-analítica de um laboratório de análises clínicas. Revista Saberes Acadêmicos, 4(1), 62-61.

Oliveira, S. A., \& Melo, N. S. 2020. Eficácia e impacto da auditoria interna na certificação de laboratórios clínicos: uma revisão integrativa. Revista Eletrônica Evidência \& Enfermagem, 6(1), 27-39.

Paula, L. N., Alves, A. R., \& Nantes, E. A. S. 2017. A importância do controle de qualidade em indústria do segmento alimentício. Revista Conhecimento Online, 9(2), 78-91.

Pasquini, N. C. 2018. Implantação de sistema de qualidade (PALC) em laboratório clínico: um estudo de caso. Revista Tecnológica da Fatec Americana, 6(1), 82-94.

Santos, A. P., \& Junior, G. Z. 2015. Controle de qualidade em laboratórios clínicos. Revista Uningá, 45, 60-67.

Santos, C. S. S., Barbosa, T. C. S., Neto, J. A. R. F., Melo, C. A., Aarão, T. L. S., \& Silveira, M. A. 2020. Controle de qualidade no Laboratório de Análises Clínicas na Fase Analítica: A Segurança dos Resultados. Brazilian Journal of health Review, 3(4), 8512-8523.

Shcolnik, W., Galoro, A., Lorencin, M., Donini, R. N., \& Jeronimo, D. 2019. Resultados de exames laboratoriais não acessados em laboratórios privados brasileiros. Jornal Brasileiro de Patologia e Medicina Laboratorial, 55(6), 641-658.

Silva, A. P., \& Steckert, C. 2019. Aplicabilidade de ferramentas da qualidade no controle de falhas do laboratório clínico. Revista Inova Saúde, 9(1), 115-128.

Sociedade Brasileira de Patologia Clínica/Medicina Laboratorial [SBPC/ML]. 2021. Norma PALC Programa de Acreditação de Laboratórios Clínicos. Ed. 2021. Disponível em http://www.sbpc.org.br/programas-da-qualidade/documentos-do-palc/.

Souza, R. K. L., Coan, E. W., \& Anghebem, M. I. 2020. Não conformidades na fase pré-analítica identificadas em um laboratório de saúde pública. Jornal Brasileiro de Patologia e Medicina Laboratorial, 56, 1-8.

Trevisan, M. 2017. Relato de experiência sobre as ações de regulação sanitária nos laboratórios clínicos em Palmas - TO. Revista Vigilância Sanitária em Debate, 5(3), 123-129.

Vieira, K. F., Shitara, E. S., Mendes, M. E., \& Sumita, N. M. 2011. A utilidade dos indicadores da qualidade no gerenciamento de laboratórios clínicos. Jornal Brasileiro de Patologia e Medicina Laboratorial, 47(3), 201-210.

\section{Minicurrículo}

Kariny Alves Santos. Supervisora de Unidade e Auditora Interna no Programa de Acreditação de Laboratórios Clínicos da Sociedade Brasileira de Patologia Clínica/Medicina Laboratorial - PALC-SBPC/ML. Possui mais de seis anos de experiencia em laboratórios de análises clínicas. Bacharelanda em Farmácia, cursando o último ano do curso de Farmácia pela Faculdade de Palmas - FAPAL.

Márcio Trevisan. Doutorando em Ciências do Ambiente pela Universidade Federal do Tocantins. Mestre em Ciências Biológicas (Bioquímica Toxicológica) pela Universidade Federal de Santa Maria. Especialista em Gestão de Vigilância Sanitária e Especialista em Regulação em Saúde no SUS pela Fundação de Ensino e Pesquisa Sírio Libanês. Graduado em Farmácia e Bioquímica pela Universidade Federal de Santa Maria. Possui experiência como farmacêutico na Secretaria Municipal de Saúde de Palmas. Atua como Analista de Saúde/Inspetor Sanitário no Núcleo de Educação em Vigilância Sanitária da Vigilância Sanitária de Palmas. Atua como docente de graduação acadêmica desde 2006. Atualmente é membro do Núcleo Estadual de Segurança do Paciente do Estado do Tocantins. 
Como citar: Santos, K.A., \& Trevisan, M. 2021. A importância do controle de qualidade nos laboratórios de análises clínicas - uma revisão integrativa. Pubsaúde, 6, a168. DOI: https://dx.doi.org/10.31533/pubsaude6.a168

Recebido: 24 abr. 2021.

Revisado e aceito: 19 mai. 2021.

Conflito de interesse: os autores declaram, em relação aos produtos e companhias descritos nesse artigo, não ter interesses associativos, comerciais, de propriedade ou financeiros que representem conflito de interesse.

Licenciamento: Este artigo é publicado na modalidade Acesso Aberto sob a licença Creative Commons Atribuição 4.0 (CC-BY 4.0). 